\title{
The Economic Mobility in Money Transfer Models
}

\author{
Ning Ding, Ning Xi and Yougui Wang* \\ Department of Systems Science, School of Management, Beijing Normal \\ University, Beijing, 100875, People's Republic of China
}

\begin{abstract}
In this paper, we investigate the economic mobility in four money transfer models which have been applied into the research on wealth distribution. We demonstrate the mobility by recording the time series of agents' ranks and observing their volatility. We also compare the mobility quantitatively by employing an index, "the per capita aggregate change in log-income", raised by economists. Like the shape of distribution, the character of mobility is also decided by the trading rule in these transfer models. It is worth noting that even though different models have the same type of distribution, their mobility characters may be quite different.
\end{abstract}

Key words: Economic Mobility, Wealth Distribution, Transfer Model

PACS: 89.65.Gh, 87.23.Ge, 05.90.+m

\section{Introduction}

The issue of income or wealth has been one of most popular topics among economists all along. With the study on this issue, the performance and wellbeing of an economy can be shown. So far, an enormous body of work has been devoted to the two aspects of this issue: distribution $[1,2,3,4,5]$ and mobility $[6,7,8]$. The former is the information that one could use to determine how many economic agents have each level of income or wealth. The latter is the phenomenon that the agents' income or wealth varies in economy over time. It is obvious that the distribution is static by nature, while the mobility is dynamic.

\footnotetext{
* Corresponding author.

Tel.: +86-10-58807876; Fax:+86-10-58807876.

E-mail address: ygwang@bnu.edu.cn (Y. Wang)
}

Preprint submitted to Elsevier Science $\quad 5$ September 2018 
The study can be traced back more than 100 years ago. An Italian economist, Vilfredo Pareto, declared that the income distributions in several European countries uniformly follow Power laws [1]. He believed that the income distribution is considerably stable both over time and across countries and there must be an uniform mechanism which governs the shape and formation of distribution. Benoit Mandelbrot observed that the distribution of income converges to Pareto distribution for many different definitions of income, for example income could be written as the sum of agricultural, commercial or industrial incomes; in cash or in kind and so on [2]. Based on the description and shape of income distribution, the measurement methods of the inequality or well-being were discussed $[9,10]$.

When the study went further, economists began to realize that only analysis on the distribution is not sufficient especially when exploring the generating mechanism of income and measuring the degree of inequality $[11,12]$. When we compare the degree of inequality in two economies with the same distribution but different dynamics, it is obvious that the static analysis based on the data of a given moment cannot provide a complete picture, and the dynamic analysis is needed. Consequently, the study on the income mobility, which is one important aspect of the dynamics, got started $[6,7,8]$.

So far, the economists devoted their efforts much more to the concept, measurement and empirical research on this issue, but a few to the mechanism behind the distribution and mobility. Several models were constructed to explain the shape of the distribution by introducing the relationship between the wages and the abilities $[3,4,5]$. On the other topic, G. C. Loury tried to employ the stochastic theory to research the mobility among successive generations of workers [13]. The lack of deep investigation in this field dose not suit with the significance of this issue, which calls for more efforts.

When physicists forayed into the economic domain with their advanced tools, they concerned the statistical characteristics of some economic variables, such as return in financial markets, volatility of GDP. Recently, researchers turn to carry out some empirical work on the income or wealth distributions and its growth or variation $[14,15,16,17,18]$. Comparing with this aspect, the more important and suitable landing zone for physicists is the mechanism behind phenomena.

Many efforts had been put into exploring the formation mechanism of distribution $[19,20,21]$. Besides, a series of multi-agent models had been constructed in which the interaction between agents is simplified as random transfer of money in a closed system $[22,23,24,25,26]$. Some of these works are reviewed in a popular journal [27], and the related models are called as money transfer models [28]. The basic model was built by A. Drăgulescu and V.M. Yakovenko basing on the analogies between money transfer in trading and energy transfer 
in molecule collision [23]. The simulation results show that the distribution of money obeys a Boltzmann-Gibbs law. A group led by B.K. Chakrabarti introduced the saving behavior into the model [24,25], and found the money distribution follows a Gamma law when all the agents are set with the same saving factor, but changes to a power law as the saving factor is set randomly. N. Ding et al introduced the preferential behavior into the trading process and obtained a stationary power-law distribution [26].

Indeed, analyzing the relationship between the distribution and the trading rule is helpful to investigate the mechanism. In fact, distribution is just one of the windows through which how the economy works can be observed. In money transfer models, where the economy is assumed to be closed, continuous transfer of money among agents leads to stationary distribution of money. However, agents still switch their positions or ranks even after the distribution gets steady. Thus, in order to explore the mechanism behind the distribution, static analysis on distribution is not sufficient. Suppose two cases where the steady distributions are observed to be the same, but their fluctuation modes of agents' ranks are different. To distinguish them, the analysis on mobility is in need, for it can provide more information as to the mechanism behind the distribution due to its dynamic and onymous nature.

Moreover, the study on mobility in the proposed models makes the evaluation criteria more complete. The aim of econophysicists to develop these models is to mimic the real economy by abstracting its essence. We cannot judge whether such abstraction is reasonable or not depending on the shape of distribution only. In other words, even though the distribution resulted from the proposed model is close to the reality, we are not convinced that it is reasonable. The mechanism might yield a different mobility character from the reality. Thus, we must take mobility into account when constructing a "right" multi-agent model. For instance, to develop a model to simulate the Japanese income distribution, the theoretical prediction on mobility must be tested against the empirical results in Ref. [18].

In addition, the transfer models proposed by econophysicists are also available for understanding the formation of mobility. Especially, these models embody the essential character of mobility, for agents in the assumed economy always change their position by exchanging the money. This phenomenon has been referred to in Hayes's review article [27].

In this paper, the mobility in four typical transfer models is observed and analyzed. In the next section, we make a brief review of the researches of economists on the mobility by which our work can be erected and from which the measurement index we employed comes. In Section 3, the four models and simulations are introduced. The results are analyzed and compared in Section 4. The final section summarizes. 


\section{Income Mobility}

Since the 1960s, economists gradually realized static snapshots of income distribution alone is not sufficient for meaningful evaluation of wellbeing and the income mobility must also be weighted. Kuznets [11] once declared that two societies "... may differ greatly in meaning because of different degrees of internal mobility ...", even if they have identical size distributions of income. And, Jarvis and Jenkins [12] argued:" To some people, greater inequality at a point in time is more tolerable if accompanied by significant mobility...".

That can be understood easily from a simple example. Suppose in an economy there are two individuals with income $\$ 1$ and $\$ 2$ initially. At the next moment, their incomes change to $\$ 2$ and $\$ 1$ respectively. The distribution in this case is unchanged, but the rank of either individual varies over time. Although the system seems unequal at either of the two moments in terms of the distribution, the fact is that the two individuals are quite equal combining these two moments. Besides, from the simple example, it can also been found out that the structure of economy may varies enormously with an unchanged distribution. So, the investigation on mobility is helpful to the measurement on equality. It should be noted that mobility is not income's particular character. There are also mobilities in some other economic concepts, such as wealth, consumption, labor and etc. Thus, the research on income mobility is also helpful to the studies on those aspects.

In contrast to the meaning of mobility research, there is less consensus on the concept and measurement. Some researchers view mobility as a reranking phenomenon: people just switch their position. To measure this purely relative mobility, some indexes are used, such as the coefficient of rank correlation, the average jump in rank and the mean number of absolute ranks changed. In the view of other researchers, any change of individuals' income leads to mobility. Here, it is an absolute concept. Naturally, the income mobility can be decomposed into two parts: one induced by reranking phenomenon and the other induced by distributional change. To measure this absolute mobility, G. S. Fields and E. A. Ok started from a series of axioms and chose an index whose expression is $l=\frac{1}{N} \sum_{k=1}^{N}\left|\log x_{k 0}-\log x_{k 1}\right|$, where $x_{k 0}$ and $x_{k 1}$ are the initial income and final income of agent $k$ respectively [29]. This index, named as "per capita aggregate change in log-income", is actually one kind of distance between two states of an economy. The "distant" index not only can reflect the change of income in aggregate level, but also is sensitive to the individuals' switch in rank.

P. V. Kerm went further and divided the mobility induced by distributional change into two parts: dispersion and growth [30]. Moreover, the empirical

analysis in the same work shows that income mobility are essentially due to 
'reranking' mobility. In fact, the 'reranking' mobility can be deemed as the transfer of income among people with given aggregate income. By identifying the income with money, this kind of the 'reranking' phenomenon can be demonstrated in the money transfer models where the total amount of money is conserved. Thus the mechanism of mobility can be analyzed based on these models that will be introduced in the next section.

\section{Transfer Models and Simulations}

The construction of transfer models provides a powerful tool for the study on the income or wealth distribution and mobility. The economies assumed in these models are pure monetary systems which are only composed of agents and money. The money is held by agents individually. And the agents exchange money to each other according to the trading rule which ensures the nonnegativity of any agent's money and the conservation of the total money. The simulation results show that no matter how uniformly and forcefully one distributes money among agents initially, the successive trades eventually lead to a steady distribution of money. And the shape of money distribution is only determined by the trading rule. There exist considerable transfer models which are used in the study on distribution. From these models, we choose the following four typical ones to show the mobility phenomena. Since the scale and the initial distribution have no effect on the final result, all of the simulations of these four models were carried out with $N=1,000$ agents and $M=100,000$ units of money. The amount of money held by each agent is set to be $M / N=100$ at the beginning.

\subsection{Model I: Ideal Gas-like Model}

By identifying money in a closed economy with energy and the trade between agents with two-body elastic collision in an ideal gas, A. Drăgulescu and V.M. Yakovenko proposed the ideal gas-like model. In each round of this model, two agent $i, j$ are chosen randomly to take part in the trade. As to which one is the payer or receiver, it is also decided randomly. Supposing the amounts of money held by agent $i$ and $j$ are $m_{i}$ and $m_{j}$ respectively, the amount of money to be exchanged $\Delta m$ is then expressed as follows:

$$
\Delta m=\frac{1}{2} \varepsilon\left(m_{i}+m_{j}\right)
$$

where, $\varepsilon$ is a random number from zero to unit. If the payer cannot afford the payment, the trade is cancelled. 


\subsection{Model II \&3 III: Uniform Saving Rate Model and Diverse Saving Rate Model}

B.K. Chakrabarti etc. thought the gap between reality and the ideal gaslike model is too huge. Some features, such as saving behavior, should be considered. They argued that the people always keep some of money in hand as saving when trading. So, B.K. Chakrabarti etc. developed the ideal gas-like model by introducing the saving behavior.

They employed the trading pattern of ideal gas-like model that two agents are chosen out to participate in the trading in each round at random. The difference is that agents keep a part of money as saving as they participate in the trade. And the ratio of saving to all of the money held by one agent is denoted by $s$ and called saving rate in this paper. The saving rates of all the agents are set before the simulations. Suppose that at $t$-th round, agent $i$ and $j$ take part in trading, so at $t+1$-th round their money $m_{i}(t)$ and $m_{j}(t)$ change to

$$
m_{i}(t+1)=m_{i}(t)+\Delta m ; m_{j}(t+1)=m_{j}(t)-\Delta m ;
$$

where

$$
\Delta m=(1-s)\left[(\varepsilon-1) m_{i}(t)+\varepsilon m_{j}(t)\right] ;
$$

and $\varepsilon$ is a random fraction between zero and unit. It can be seen that if $\Delta m$ is positive, agent $i$ is the receiver of the trade, otherwise the payer. This model degenerates into the ideal gas-like model if $s$ is set to be zero. In this model, all of agents are homogenous with the same saving rate. So we call it uniform saving rate model.

B.K. Chakrabarti etc. modified the model by introducing diverse saving rate. They set agents' saving rates randomly before the simulations and keep them unchanged all through the simulations. Likewise, this model is called diverse saving rate model. Correspondingly, the trading rule Equation (2) is transformed into

$$
m_{i}(t+1)=m_{i}(t)+\Delta m ; m_{j}(t+1)=m_{j}(t)-\Delta m ;
$$

where

$$
\Delta m=\left(1-s_{i}\right)(\varepsilon-1) m_{i}(t)+\left(1-s_{j}\right) \varepsilon m_{j}(t) ;
$$

and $s_{i}, s_{j}$ are the saving rates of agent $i$ and $j$ respectively. 


\subsection{Model IV: Preferential Dispensing Model}

We attribute two forces to the formation of power-law distribution: one is preference in dispensing, which lets the rich have higher probability to get the money; the other one is stochastic disbursement, which ensures that the rich would be deprived of wealth and become the poor in some day. In order to verify this idea, we proposed a new money transfer model named as preferential dispensing model and obtained a steady power-law distribution of money.

In each round of this model, every agent pays money out, and the ratio of payment to the amount of money he held is determined randomly. To any unit of these money, a receiver is chosen randomly from the agents except the payer. The probability $p_{i, j}$ at which agent $i$ (potential receiver) gets the money from agent $j$ (payer) is given by

$$
p_{i, j}=\frac{m_{i}}{\sum_{n=1(n \neq j)}^{N} m_{n}},
$$

where $m_{i}$ is the amount of money held by agent $i$ before the trade. The constraint $n \neq j$ in this rule eliminates the possibility for the payer to get back the money he has paid out.

\section{Results and Discussion}

To show the 'reranking' mobility, at the end of each round, we sort all of agents according to their money and record their ranks. Here, we choose the rank rather than the amount of money to show the mobility because the fluctuation of rank can reflect the mobility especially the 'reranking' mobility with an unchanged distribution. Another reason is that the former is too sensitive to the perturbation. In these transfer models, agents keep to switch their rank over time whether the distribution is stationary or not. To avoid the effect of transients, all of data are recorded after the money distributions get stationary. It should be noted that the time intervals of sampling are different for these models as collecting the data. The data are sampled every 1000 rounds ${ }^{1}$ in the first three models and every round in model IV. We know that only a pair of agents take part in trade in each round of the first three models, while all of agents do so in the Model IV. It is obvious that the mobilities are not in the same level if the sampling intervals are the same in these models. In order to make the mobilities comparable, we proceed sampling in the way mentioned above, for the times per agent taking part in trading in one round

$\overline{1}$ Which is identical to the number of agents $N$. 
in model IV are equal to those in 1000 rounds in the first three models. In this way, we get the time series of rank for all agents. Some typical time series of rank from the sampling data of these four models are shown in Figure 1 correspondingly. The rank is inversely related to the amount of money held, so the more money an agent held, the lower his curve is in these figures.

From the time series of ranks plotted in Figure 1, we can compare the character of rank fluctuation of these four models with each other. It can be seen that the fluctuations in model I and model II are quite similar except the frequency(Fig.1a and 1b). All of the agents can be the rich and be the poor. The rich have the probability to be poor and the poor also may be luck to get money to be the rich. In other words, the economies in these two models are quite fair where every agent is equal in the opportunity. As mentioned in Section 3 , the model I is a special case of model II when the uniform saving rate is zero. Thereby the Figure $1 \mathrm{a}$ and $\mathrm{b}$ can be taken as the results of the cases where the uniform saving rate are 0 and 0.5 respectively. Comparing them, it can be seen that the lower the saving rate, the higher fluctuation frequency, which means lower saving rate leads to greater mobility in model II. From Fig.1c, we can see that the economy in model III is highly stratified where the rich always keep their position, and the poor are doomed to being the poor. Moreover, the agents in different level differ in the rank fluctuation. The higher the agent's ranks, the smaller the variance of his ranks. From Fig.1d, each of the agents has the same probability to be the rich in the model IV just like model I. However, the time interval that agents keep in their current position is quite longer, which results in a lower degree of mobility.

Now we turn to quantitative measurement of mobility. Although there are quite a few indexes for measuring the relative mobility, they fall into disuse due to various deficiencies. In contrast, the measurement index raised by G. S. Fields et al has the advantage over them. It can be applied for not only the absolute mobility but also the relative one, as long as we take the agents' ranks as the sampling variable. So we define the 'reranking' mobility between the two samples recorded at different moments as follows:

$$
l\left(t, t^{\prime}\right)=\frac{1}{N} \sum_{i=1}^{N}\left|\log \left(x_{i}(t)\right)-\log \left(x_{i}\left(t^{\prime}\right)\right)\right|,
$$

where $x_{i}(t)$ and $x_{i}\left(t^{\prime}\right)$ are the ranks of agent $i$ at $t$ and $t^{\prime}$ respectively. It is obvious that the bigger the value of $l$, the greater the level of mobility. The values of $l$ of the four models are shown in Table 1 . These results are gotten in the following way. Firstly, more than 9000 samples are recorded continuously after the distribution gets steady. Secondly, the 'distance' between any two consecutive samples is reckoned. Finally, the average of these 'distances' is calculated as $l$. Just as mentioned above, in the first three models, the 
difference between $t$ and $t^{\prime}$ is 1000 , while that in model IV is 1 .

The data given in Table 1 verify our primary conclusion about model I and II derived from Fig.1a and 1b in some degree: the mobility decreases when the saving rate increases. The intuition for this result is straightforward. The more money agents keep when trading, the less money they pick out to participate in the trade. And so the less probability of change in rank. Therefore, the higher saving rate, the lower mobility.

Comparing with model II, the value of mobility is quite small in model III. This is obviously due to the stratification. However, what surprised us is that the value is much smaller than that in model II when the uniform saving rate is 0.5 , even than that when the uniform saving rate is set to be 0.9 . As we know, the agents in the economy assumed interact with each other and the index cannot be obtained by simple mathematical averaging. As the agents with high ranks almost do not change their positions, their contribution to the mobility is nearly none. Although the rest of agents move drastically, the range of their rank fluctuation is limited. As a result, the mobility in model III is very small. With regard to stratification, the diverse saving rates must be culprit because they are the only denotation of the agents' heterogeneity. To demonstrate this point, we show the relation between the agents' ranks and their saving rates in Fig.2. It can be seen clearly that there exists the negative relation between the rank and the saving rate. And the rank fluctuation of agents with low saving rate is more drastic than that of agents with high saving rate.

Now, we turn to the mobility in Model IV. From Table 1, we can see that no stratification appears in this case and the degree of mobility is the smallest. The homogeneity of agents' behavior pattern in this model gives every agent an equal chance to get rich. However, the preferential behavior ensures the agents keeping their position for a relatively long period. As a result, the assumed economy evolves with very low mobility and without stratification. Comparing model III and model IV, we can conclude that although both of the monetary distributions follow Power laws, their mobility characters are totally different.

From the above discussion, it can be seen that the index can help us to compare the mobility quantitatively. However, the index itself can not give the whole picture of mobility. The "distant" index we used in this paper can be rewritten as

$$
l\left(t, t^{\prime}\right)=\frac{1}{N} \sum_{i=1}^{N}\left|\log \left(\operatorname{vol}_{i}\left(t, t^{\prime}\right)+1\right)\right|,
$$

where $v_{i} l_{i}\left(t, t^{\prime}\right)$ actually denotes the volatility of agent $i$ 's rank. It takes the 
following form

$$
\operatorname{vol}_{i}\left(t, t^{\prime}\right)=\frac{x_{i}\left(t^{\prime}\right)-x_{i}(t)}{x_{i}(t)}
$$

The new expression indicates that the index is a presentation of volatility of rank at aggregate level. To examine the distribution of volatility can provide us the information of mobility in more detail.

The distributions of the rank volatility for the four models are illustrated in Figure 3 respectively. It is noted that the distributions in model I and II are quite similar and their right tails follow power laws. In model II, we further calculated the exponent of the power-law distribution $p(x) \propto x^{\alpha}$ for different uniform saving rates. As shown in Fig.4, the exponent $\alpha$ varies with the saving rate in an exponential way. This result is consistent with the dependence of the mobility index on the saving rate. When the saving rate gets higher, the money exchanged will be less and the volatility of rank will decrease. Consequently, when the saving rate increases, the right side of volatility distribution will shift to the vertical axis, leading to a more steeper tail. From Figure 3c and $3 \mathrm{~d}$, it is clear that the distributions of the rank volatility for models III and IV are quite different. The tail of volatility distribution of model III converges to an exponential line as the times of simulations increase. In model IV, the part of the volatility distribution within the range from 0 to about 1.5 has a good fitting to an exponential line, but the tail scatters no matter how many times of the simulations are performed.

\section{Summary}

To economists, mobility is a supplement to distribution when the equalities are compared. To physicists, it is an approach to the mechanism behind the distribution in microscope. The purpose of this paper is to investigate the mobility phenomena in four typical money transfer models. Some characters of mobility are also presented by recording the time series of agents' ranks and observing the distribution of rank volatility. We find that the mobility is also decided by the trading rule in these transfer models. Our finding implies that the economies with the same distribution may be different in mobility. It would be helpful to take the character of mobility into account when constructing a multi-agent model on the issue of distribution. 


\section{Acknowledgement}

We thank Prof. Zengru Di for his help discussions and comments. The work is partially supported by NSFC under grant No.73071072.

\section{References}

[1] V. Pareto, Cours d'Economie Politique, Macmillan, Paris, 1897.

[2] B. Mandelbrot, Int. Econ. Rev. 1(2), May, 79 (1960).

[3] A. D. Roy, Oxford Economic Papers 3(2), June, 135 (1951).

[4] C. N. Teulings, J. Polit. Econ. 103(2), April, 280 (1995).

[5] D. G. Champernowne, Econ. J. LXIII, June, 318 (1953).

[6] B. R. Schiller, Am. Econ. Rev. 67(5), 926 (1977).

[7] A. F. Shorrocks, Economica 50, 3 (1983).

[8] G. S. Feilds and E. A. Ok, in Handbook of Inequality Measurement, edited by J. Silber, Kluwer Academic Publishers, Dordrecht, 2003. p. 557-596.

[9] A. Sen, in Ethical Measurement of Inequality: Some Difficulties, edited by W. Krelle and A. F. Shorrocks, Nothholland Publishing Company, Nwtherlands, 1977. p. 81-94.

[10] F. A. Cowell, Rev. Econ. Stud. XLVII(3), April, 521 (1980).

[11] S. S. Kuznets, Modern Economic Growth: Rate, Structure and Spread, Yale University, New Haven, 1966. p.203.

[12] S. Jarvis and S. P. Jenkins, Econ. J. 108, 1 (1998).

[13] G. C. Loury, Econometrica 49(4), 843 (1981).

[14] W. Souma, Fractals 9, 463 (2001).

[15] M. Levy and S. Solomon, Physica A 242, 90 (1997).

[16] A. Drăgulescu and V. M. Yakovenko, Eur. Phys. J. B 20, 585 (2001).

[17] A. Drăgulescu, and V. M. Yakovenko, Physica A 299, 213 (2001).

[18] Y. Fujiwara, W. Souma, H. Aoyamac, T. Kaizoji, M. Aoki, Physica A 321, 598 (2003).

[19] M. Levy and S. Solomon, Int. J. Mod. Phys. C 7, 595 (1996).

[20] J.-P. Bouchaud and M. Mezard, Physica A 282, 536 (2000). 
[21] F. Slanina, Phys. Rev. E 69, 046102 (2004).

[22] S. Ispolatov, P. Krapivsky, and S. Redner, Eur. Phys. J. B 2, 267 (1998).

[23] A. Drăgulescu and V. M. Yakovenko, Eur. Phys. J. B 17, 723 (2000).

[24] A. Chakraborti and B. K. Chakrabarti, Eur. Phys. J. B 17, 167 (2000).

[25] A. Chatterjee, B. K. Chakrabarti and S. S. Manna, Physica A 335, 155 (2004).

[26] N. Ding, Y. Wang, J. Xu and N. Xi, Int. J. Mod. Phys. B 18(17-19), 2725 (2004).

[27] B. Hayes, Am. Scientist 90, 400 (2002).

[28] Y. Wang, N. Ding, N. Xi, To appear in Practical Fruits of Econophysics, edited by H. Takayasu, Springer-Verlag, Tokyo, 2005.

[29] G. S. Fields and E. Ok, Economica 66, 455 (1999).

[30] P. V. Kerm, Economica 71, 223 (2004). 

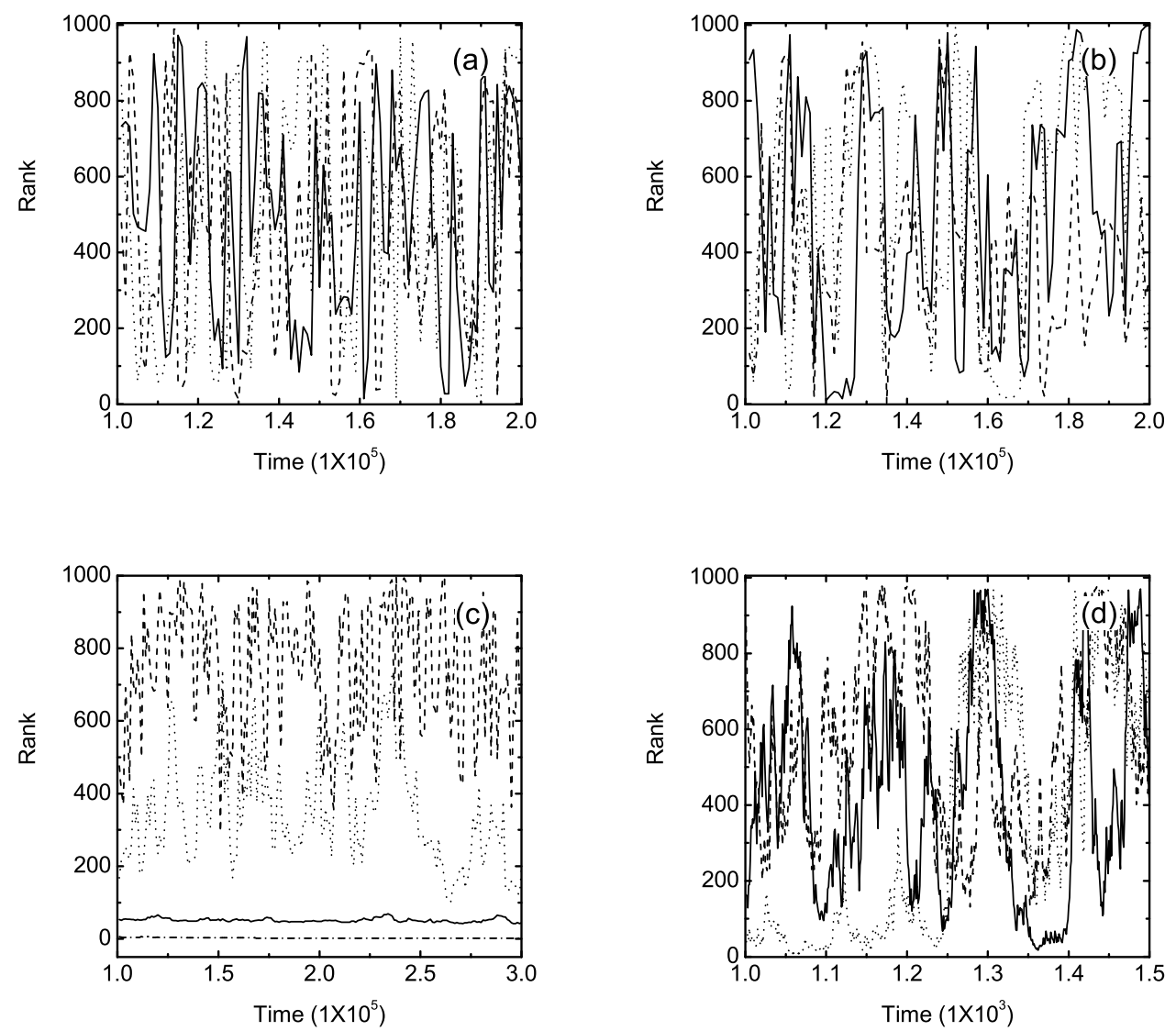

Fig. 1. The typical time series of ranks from model I: Ideal Gas-like Model (a), model II: Uniform Saving Rate Model with saving rate 0.5 (b), model III: Diverse Saving Rate Model where the saving rates of these typical agents are 0.99853, 0.9454, 0.71548 and 0.15798 (from bottom to top respectively) (c) and model IV: Preferential Dispensing Model (d). 


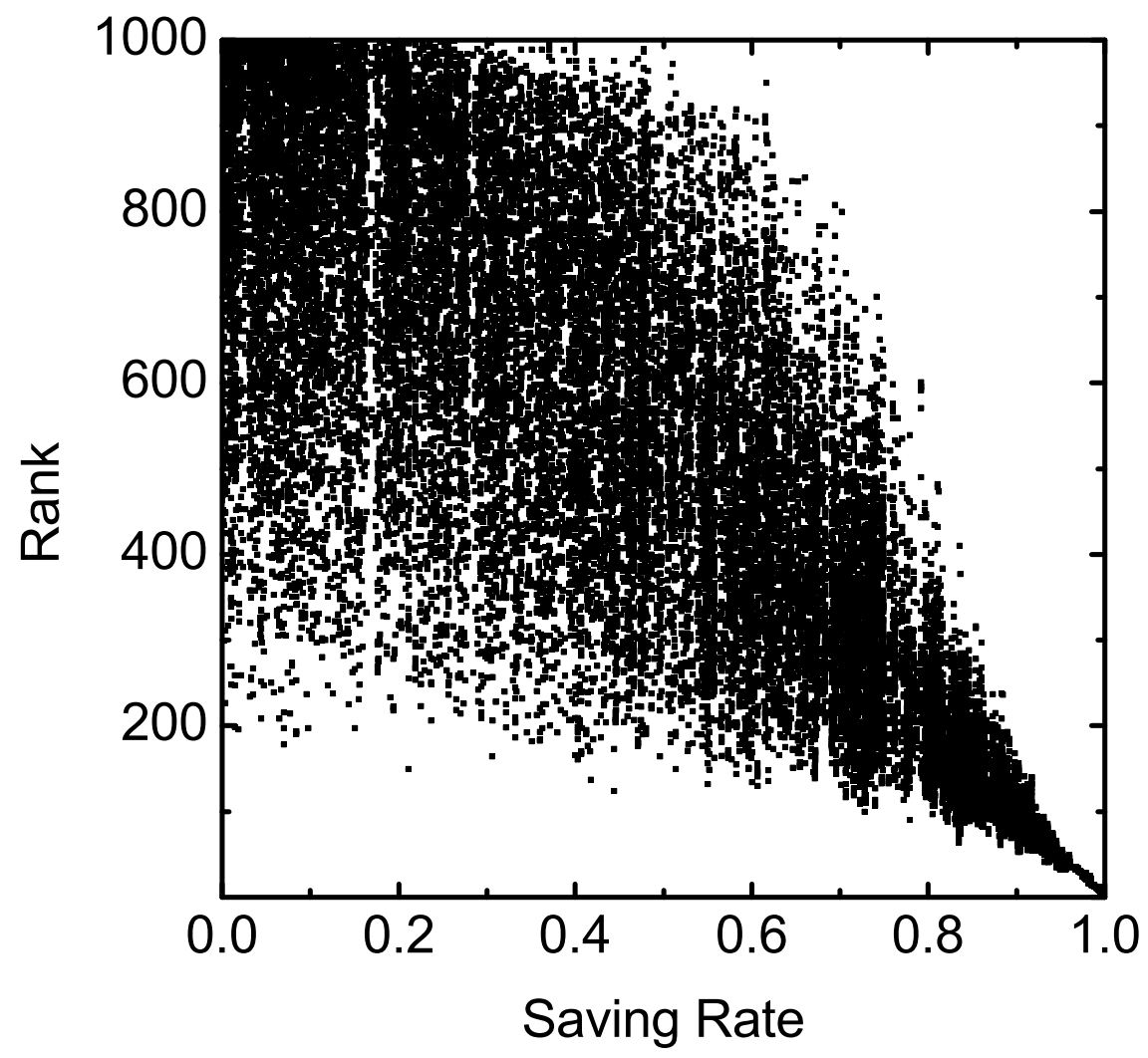

Fig. 2. The correlation between agents' ranks and saving rate in model III. 

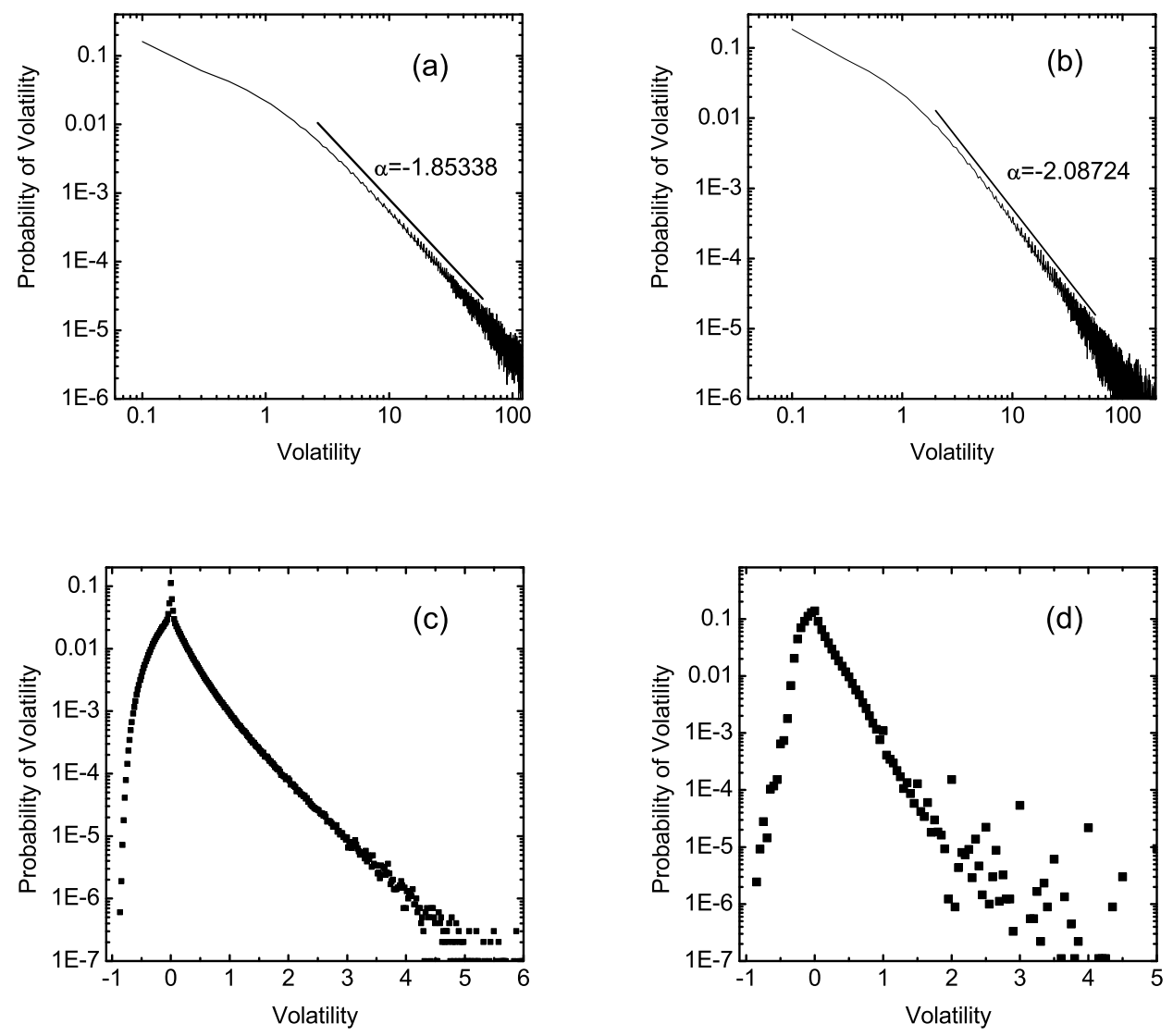

Fig. 3. The distribution of the volatility of agents' ranks from model I: Ideal Gas-like Model (a), model II: Uniform Saving Rate Model with saving rate 0.5 (b), model III: Diverse Saving Rate Model (c) and model IV: Preferential Dispensing Model (d). 


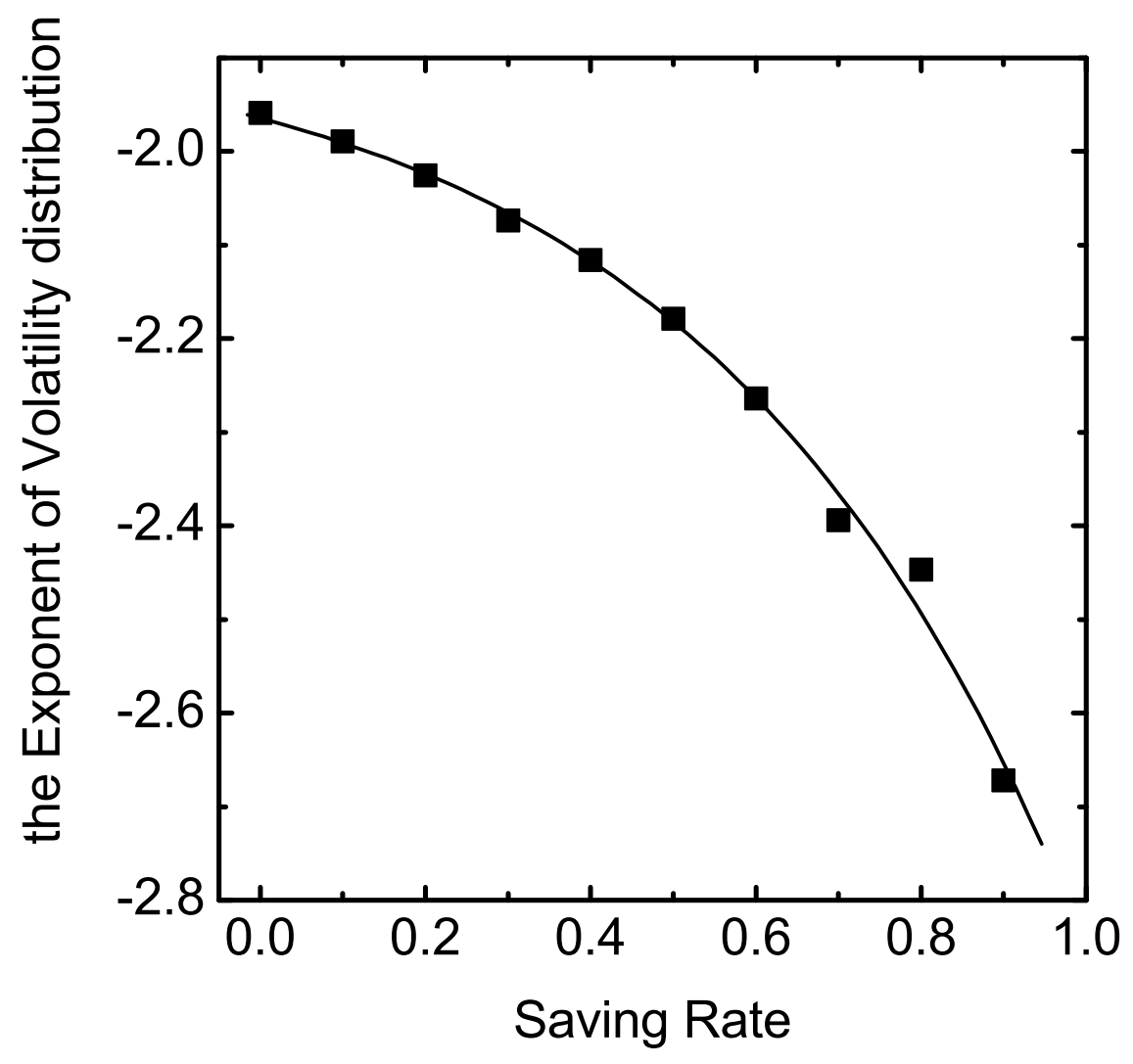

Fig. 4. The relation between the uniform saving rate and the slope of the volatility distribution in model II: simulation results(dots) and fitness result(curve). $\alpha=-0.104 e^{s / 0.443}-1.860$ 
Table 1

Comparison of Four Typical Transfer Models With Respect to Monetary Distribution and Mobility

\begin{tabular}{c|c|c|c}
\hline & Monetary Distribution & Mobility $l\left(t, t^{\prime}\right)$ & Stratification \\
\hline Model I & Exponential Law & 0.72342 & No \\
\hline Model II & Gamma law & & No \\
$s=0.1$ & & 0.70269 & \\
$s=0.3$ & & 0.65165 & \\
$s=0.5$ & & 0.58129 & \\
$s=0.7$ & & 0.4773 & \\
$s=0.9$ & & 0.30212 & \\
\hline Model III & & 0.19671 & Yes \\
\hline Model IV & Power Law & 0.14828 & No \\
\hline
\end{tabular}

